$N=2(n-2+2 p)$ is the number of pinch points on a ruled surface in $S_{3}$ and if $t=2$, then $N=3(n-4+4 p)$ is the number of inflexional tangent lines to a ruled surface in $S_{5}$. We also see that a $V_{3}^{n}$, given by $k=3$, has $2(n-3+3 p)$ pinch points if it in $S_{5}$ and $3(n-6+6 p)$ inflexional tangent lines if it is in $S_{8}$; and so forth.

The University of California

\title{
CLASS NUMBER IN A LINEAR ASSOCIATIVE ALGEBRA*
}

BY GRACE SHOVER

1. Introduction. In this paper the finiteness of the class number is established for every division algebra taken over the rational field. For every semi-simple algebra, the right and left class numbers are proved to be equal. The classical method for proving the finiteness of the class number in algebraic fields depends upon the multiplication of ideals, but the problem is treated in this paper for the general case without reference to the concept of ideal multiplication. The finiteness of the class number for every algebraic field follows as a special case.

2. Definitions and References. Algebra, domain of integrity, and ideal are defined as in a previous paper. $\dagger$

The norm of an ideal $\Omega$ is defined to be the absolute value of $|G|$, where $G$ is a matrix representing $\Omega . \ddagger$

A necessary and sufficient condition that a matrix $G$ represent a left (right) ideal is that

$$
G R_{p}^{\top}=D_{p} G,\left(G S_{p}=Q_{p} G\right), \quad(p=1,2, \cdots, n),
$$

where $R_{p}{ }^{\top}$ is the transpose of the first matrix of $e_{p}\left(S_{p}\right.$ is the second matrix of $\left.e_{p}\right)$. The matrices $D_{p}\left(Q_{p}\right)$ are called the class matrices corresponding to the ideal matrix $G$.

\footnotetext{
* Presented to the Society, November 28, 1931.

† Shover and MacDuffee, this Bulletin, vol. 37 (1931), pp. 434-438.

$\ddagger$ MacDuffee, Transactions of this Society, vol. 31 (1929), pp. 71-90.
} 
Every principal left (right) ideal may be represented by the second (transpose of the first) matrix of an integer of the albra. Every such matrix represents a left (right) ideal.

Two non-singular left (right) ideals $\Omega_{\alpha}$ and $\Re_{\beta}$ are said to be equivalent if there exist integers of the domain $\sigma_{1}$ and $\sigma_{2}$ such that

$$
\Re_{\alpha} \sigma_{1}=\Re_{\beta} \sigma_{2}, \quad\left(\sigma_{1} \Re_{\alpha}=\sigma_{2} \Re_{\beta}\right) .
$$

In matric notation,

$$
G_{\alpha} S\left(\sigma_{1}\right)=A G_{\beta} S\left(\sigma_{2}\right),\left(G_{\alpha} R^{\top}\left(\sigma_{1}\right)=A G_{\beta} R^{\top}\left(\sigma_{2}\right)\right),
$$

where $|A|= \pm 1$. If $\Re_{\alpha}$ and $\mathfrak{\Omega}_{\beta}$ are two equivalent non-singular left (right) ideals represented, respectively, by $G_{\alpha}$ and $G_{\beta}$, then the corresponding class matrices $D_{\alpha p}$ and $D_{\beta p}\left(Q_{\alpha p}\right.$ and $\left.Q_{\beta p}\right)$ are similar, that is,

$$
A D_{\alpha p}=B_{\beta p} A,\left(A Q_{\alpha p}=Q_{\beta p} A\right),
$$

where $|A|= \pm 1$, and conversely. If $D_{\alpha p}=D_{\beta p},\left(Q_{\alpha p}=Q_{\beta p}\right), G_{\alpha}$ and $G_{\beta}$ are said to belong to the same minor class. The left (right) class number is the number of non-equivalent non-singular left (right) ideals in the algebra.

It has been shown* that if $T$ is the discriminant matrix of an algebra, the $R$ and $S$ matrices of a number are connected by the relation $S T=T R$.

3. Class Number of Left Ideals. Suppose

$$
\beta=\sum b_{i} e_{i}, e_{1}=1,
$$

is a number of the left ideal $\Re_{\alpha}$ with basis $\alpha_{1}, \alpha_{2}, \cdots, \alpha_{n}$. Then

$$
\beta=\sum m_{1 i} \alpha_{i},
$$

where the $m_{1 i}$ are rational integers. Multiplying by $e_{r}$ on the left, we have

$$
e_{r} \beta=\sum m_{1 i} e_{r} \alpha_{i},
$$

Since the $\alpha_{i}$ form a basis for a left ideal there exist rational integers $m_{r i}$ such that

$$
e_{r} \beta=\sum m_{r i} \alpha_{i},
$$

* MacDuffee, Transactions of this Society, vol. 33 (1931), pp. 425-432. 
or

$$
\sum b_{i} e_{r} e_{i}=\sum m_{r i} a_{i s} e_{s}
$$

and

$$
\sum b_{i} c_{r i s} e_{s}=\sum m_{r i} a_{i s} e_{s} .
$$

Because of the linear independence of the basis numbers of the algebra, it follows that

$$
\sum b_{i} c_{r i s}=\sum m_{r i} a_{i s}
$$

Taking matrices, we have $S(\beta)=M G_{\alpha}$, where $M$ is an integral matrix.

Conversely, if $S(\beta)=M G_{a}$, then

$$
e_{r} \beta=\sum m_{1 i} \alpha_{i}
$$

Let $r=1$. Then

$$
\beta=\sum m_{1 i} \alpha_{i} \text {. }
$$

We have now proved the following lemma for left ideals.

LEMMA 1. If $\beta$ is a number of the left ideal $\Omega_{\alpha}$, there exists an integral matrix $M$ such that $S(\beta)=M G_{\alpha}$, and conversely.

LEMma 2. If $\beta$ is any number not a divisor of zero of the nonsingular left ideal $\Omega$, then the adjoint $M^{\mathrm{A}}$ of $M=S(\beta) G^{\mathrm{I}}$ represents a left ideal and it belongs to the same minor class as $G$.

Since $M G$ is a second matrix of the algebra, it is commutative with the transpose of every first matrix. Hence

$$
M G R_{p} \mathrm{~T}=R_{p} \mathrm{~T} M G=M D_{p} G,
$$

Cancelling the $G$ 's in the last two members, multiplying them on both left and right by $M^{\mathrm{A}}$, and dividing by $|M|$, we have

$$
M^{\mathrm{A}} R_{p}^{\mathrm{T}}=D_{p} M^{\mathrm{A}} .
$$

LEMMA 3. The determinants $\left|\Sigma h_{i} S_{i}\right|$, where the $h_{i}$ are real and $\left|h_{i}\right| \leqq 1$, have an upper bound in the algebra.

The greatest number attainable for any determinant of order $n$ is less than $n ! g^{n}$, where $g$ is the absolute value of an element of the determinant of maximum absolute value. But $g$ can not be greater than $\Sigma\left|c_{r i s}\right|$ for any $r, s$. Hence the determinant is bounded for any set of $h_{i}$ 's. 
LEMMA 4. The number of non-singular ideals whose norms are less than a given real number is finite.

The number of ideals of any given norm $g$ is finite. Applying Hermite's reduction theorem* to all matrices $G=\left(g_{r s}\right)$ of norm equal to the absolute value $g$ of $|G|$, we have

$$
\pi g_{r r}=g, \quad\left(g_{r r}>0\right) .
$$

There are a finite number of choices for the $g_{r r}$. Since all the elements are positive and we have

$$
g_{r s}<g_{r r}, \quad(r>s),
$$

and

$$
g_{r s}=0, \quad(r<s),
$$

there are a finite number of choices for all the elements in the matrix. Since there are a finite number of choices for norms less than a given real number, we have the following theorem.

Theorem 1. In a division algebra the left class number is finite.

By a theorem of Minkowski, $\dagger$ it is possible to obtain integers $x_{i},(i=1,2, \cdots, n)$, not all zero such that

$$
\left|\sum x_{i} a_{i j}\right| \leqq(|a|)^{1 / n}
$$

where $G=\left(a_{i j}\right)$ is a matrix representing the non-singular left ideal $\Omega_{\alpha}=\left(\alpha_{1}, \alpha_{2}, \cdots, \alpha_{n}\right)$ and $a=|G|$. If $\beta=\Sigma x_{i} \alpha_{i}$, then

$$
S(\beta)=M G_{\alpha} \text { and } S(\beta)=\sum x_{i} a_{i j}\left(c_{r j s}\right) \text {. }
$$

Since the algebra is a division algebra, $S(\beta)$ is non-singular and $|M| \neq 0$. Then

$$
|S(\beta)|= \pm|G| \cdot\left|\sum h_{i} S_{i}\right|
$$

where

$$
\left|h_{i}\right|=\left|\sum x_{i} a_{i j} /(|a|)^{1 / n}\right| \leqq 1 .
$$

Since $\left|\Sigma h_{i} S_{i}\right|$ is bounded by a number $b,|M|$ is also bounded by $b$ and $\left|M^{\mathrm{A}}\right| \leqq b^{n-1}$. But $M^{\mathrm{A}}$ represents a non-singular ideal and it belongs to the same minor class as $G$. Hence in every class.

* Journal für Mathematik, vol. 41 (1851), p. 193.

$\dagger$ Minkowski, Geometrie der Zahlen, Teubner, 1910, p. 104. 
it is possible to find a non-singular ideal whose norm is less than a given finite number which depends only on the algebra. Therefore the class number is finite.

4. Equality of the Two Class Numbers.

LEMMA 5. If $G$ is a left ideal matrix, then $G^{\mathrm{AT}} T^{\mathrm{A}}$ is a right ideal matrix whose class matrices are the transposes of those of $G$.

We have

$$
G R_{p}^{\top}=D_{p} G,
$$

where the $D_{p}$ are integral. Also

$$
S_{p} T=T R_{p}, \quad \text { or } \quad R_{p}^{\top}=T^{\top} S_{p}^{\top} T^{\top} .
$$

Then

$$
G T^{\top} S_{p}^{\top}=D_{p} G T^{\top}, \quad \text { or } \quad S_{p} T G^{\top}=T G^{\top} D_{p}^{\top} .
$$

Hence

$$
G^{\mathrm{AT}} T^{\mathrm{A}} S_{p}=D_{p}^{\mathrm{T}} G^{\mathrm{AT}} T^{\mathrm{A}} .
$$

THEOREM 2. In every semi-simple algebra the left class number is equal to the right class number.

Consider the left ideal matrices $G_{\alpha}$ and $G_{\beta}$ and the corresponding right ideal matrices $G_{\alpha}{ }^{\mathrm{AT}} T^{\mathrm{A}}$ and $G_{\beta}{ }^{\mathrm{AT}} T^{\mathrm{A}}$. If the $D_{\alpha p}$ are (not) similar to the $D_{\beta p}$, the $D_{\alpha p}^{\mathrm{T}}$ are (not) similar to the $D_{\beta p}{ }^{\mathrm{T}}$. Hence the right class number is not less (greater) than the left. Hence the two are equal.

Lemmas and theorems similar to those given here for left ideals may be proved for right ideals.

Columbus, Оhiо 